\title{
El infinitesimal eco del secreto: T rans Pacific Partnership, desregulación y nanotecnología en el Antropoceno
}

Rainer María Hauser Molina ${ }^{1}$

\section{Resumen}

Con una mirada que busca establecer puentes entre las ciencias y generar la reflexión del significado, este artículo entonces "política", aborda el contexto social y económico en que se desenvuelve la relación global entre regulación y nuevas tecnologías. Consideramos que la Nanotecnología y sus apuestas de futuro, están determinadas a un avance a la vez exponencial en todas las áreas y al mismo tiempo, sin control estatal, ni regulación ninguna: el capitalismo vive un período inédito de acumulación monopólica, basado en la desregulación, el descontrol. Planteamos que una prueba extrema -pero evidente- de ello, son los dos "secretos" actuales del cyberespionaje, y el Trans Pacific Partnership. Mostramos como el peso invisible de las Grandes Corporaciones que se encuentran detrás de su firma, atenta contra el Estado y su funcionamiento. En una perspectiva de "filosofía de la historia", decimos que vivimos un Renacimiento, que como el del siglo $\mathrm{XV}$, tiene dos caras: para unos despertar y conocimiento, para los otros opresión y genocidio. Postulamos que si con Copérnico, el ser humano (cultura) alcanzó su centro rodeado de la naturaleza, hoy esa relación se invierte y desde los dos extremos de lo más grande y lo más pequeño (Cambio Climático y Nanotecnología), la naturaleza está ahora en el centro de la cultura.

Palabras clave: Cambio Climático, nanotecnología, capitalismo, desregulación, cyberspyonage 


\section{The inf initesimal echo secret: T rans Pacific Partnership, deregulation and nanotechnology in the Anthropocene}

Abstract

With a look that seeks to build bridges between sciences and generate reflection of meaning, this article therefore "on policy", addresses the social and economic context in which the overall relationship between regulation and new technology develops. We believe that nanotechnology and its commitments to the future, are determined to advance exponentially in all areas and at the same time, without state control nor regulation: capitalism lives an unprecedented period of monopolistic accumulation, based on deregulation, the lack of control. We propose that an extreme test-but it obviously, are the two current "secrets" cyberespionaje, and the Trans Pacific Partnership. We show how the invisible power of large corporations, which are behind its signature, violates the state and its functioning. In a perspective of "philosophy of history", we say that we live in a Renaissance, which, as in the fifteenth century, has two faces: for some awakening and knowledge and to other, oppression and genocide. We postulate that if with Copernicus, man (culture) reached its center surrounded by nature, today this relationship is reversed and from both ends of the largest and the smallest (Climate Change and Nanotechnology), nature is now in the center of culture.

Keywords: Climate Change, nanotechnology, capitalism, deregulation, cyberspyonage. 


\section{0 infinitesimal eco do secreto: Trans Pacific Partnership, desregulação e nanotecnologia no Antropoceno}

\section{Resumo}

Com um olhar que busca estabelecer pontes entre as ciências e gerar a reflexão do significado, este artigo então "político", aborda o contexto social e econômico em que se desenvolve a relação global entre regulação e novas tecnologias. Consideramos que a Nanotecnologia e suas apostas no futuro estão determinadas a um avanço exponencial em todas as áreas e ao mesmo tempo, sem controle estatal, nem regulação nenhuma: o capitalismo vive um período inédito de acumulação monopólica, baseado na desregulação e no descontrole. Colocamos que a prova extrema -porém evidente- disto são os dois "secretos" atuais da cyberespionagem, e o Trans Pacific Partnership. Mostramos como o peso invisível das Grandes Corporações que se encontram no lugar de seu caráter atenta contra o Estado e seu funcionamento. Em uma perspectiva de "filosofia da história", dizemos que vivemos um Renascimento, que como o do século XV, tem duas caras: para alguns despertar e conhecimento, para outros opressão e genocídio. Postulamos que se com Copérnico, o ser humano (cultura) alcançou seu centro rodeado da natureza, hoje esta relação se inverte e desde os dois extremos do maior e do menor (Mudança Climática e Nanotecnologia), a natureza está agora no centro da cultura.

Palavras chave: Mudança Climática, nanotecnologia, capitalismo, desregulação, cyberspionagem 


\title{
1. La cena del Patriarca. (En memoria de 0 rígenes: "Credo qui absurdum est")
}

\begin{abstract}
Todos los métodos que ayudan a producir plusvalía, favorecen también la acumulación, todos los progresos de la acumulación, ayudan a producir plusvalía. De esto se deduce que cualquiera sea el nivel de los salarios, la condición del trabajador empeora al acumularse el capital. K. Marx (1973), El Capital. Tomo 1, p.682.
\end{abstract}

Que esta época está marcada por la complejidad, no sabríamos negar. En cualquier ámbito de atención en el cual se posara el vuelo de pájaro de nuestra conciencia, las cosas -y las no cosas-se demuestran resultado de muchas otras, en transformaciones aceleradas y constantes, y como dice Levi-Strauss, en "plétora de significantes sin significado". De allí que tal como fuera el caso de nuestro hábito humano de construir mitos para transformarlos en historia, aunque nunca pueda establecerse el punto preciso en que esa transformación de una cosa en otra ocurre ("anaclástica" la llama el maestro), vamos dejando fragmentos de construcción de savia en estos árboles de sol que siempre claman por un relato, el que pueda decirse, el que sea escuchado, el que tranquilice, el que transforme nuestra prehistoria en historia, el que finalmente y sin más, nos haga entender, para seguir nuestro vuelo de sociedades, en que "soy, en tanto somos", como dice el Zwahili "Ubuntu”, ya rescatado por la construcción de software libre.

Señalo, en ésta búsqueda de otorgar significado a las cosas y como un homenaje a las culturas de los pueblos -tan paradojalmente ausentes de estas problemáticas-, que la etimología del nombre de la ciudad que nos acoge, "Aracaju", significa "El Tucán en el Arbol”, en la lengua de los primigenios habitantes de este lugar. La coincidencia de haber estudiado sus mitos, los mitos Tupi Guaraní, con Levi-Strauss, me ha hecho entender que las propiedades de los Mitos, pueden aportar mucho para comprender significados en los desarrollos de la cybercultura y la tecno-ciencia contemporá- 
nea, como alguna vez lo recogí en otro lugar (Cyberantropología en la era del post-alfabeto, 2001), cuestión que no se imagina. Sea aquí la mención, al pueblo Tupi que nos acoge, un tributo al enorme, polisémico y aun no plenamente comprendido aporte de los pueblos originarios, cuya relación de convivencia intrínseca y necesaria con la naturaleza, se hace cada vez más comprensible para nosotros, en la medida que aumenta la velocidad en que nos alejamos de ella. Sea también esta mención, una señal a los temas de pérdida de libertades, control, secreto y falta de transparencia que abordamos y que de distintas maneras los pueblos originarios siguen sufriendo, desde hace 500 años.

Este escrito, que se situa desde el punto de vista de la "Mediología" (Debray, 2000) es decir de la confluencia entre la ciencia, los medios de difusión y el efecto en las políticas públicas, en la era del antropoceno, refiere sin duda, a una concepción del conocimiento como dimensión política. De la suerte, no puede dejar de mencionar un hecho ex post a su escritura inicial, que fue sin embargo relatado en la conferencia (nanoweb.tv). Al cabo, las teorías de la conspiración, se alimentan de hechos reales, que no podemos explicar de acuerdo a cadenas causales y que sin embargo, ocurren. Así, me encontraba en el Hotel de Aracaju, revisando mi presentación del día siguiente y a los tres minutos de estar on-line y haber abierto el archivo que había llamado "Snowden", mi netbook, colapsó, con todos sus archivos. "Missing Operative System", tintineo en la pantalla, que no dejaría de ser negra, de alli en adelante. Como no señalarlo, al cabo, si es precisamente una de las propiedades de los sistemas de control que se han hecho conocidas a través de las publicaciones de Wikileaks y The Guardian: como estamos siendo vigilados y como esa vigilancia es activa: sabemos que el sistema PRISM, que puede actuar directamente y on line, sobre las computadoras que decida y de las múltiples maneras que al poder parezca.

De allí que me sea necesario referir brevemente a esta línea de investigación -tan pobre de resultados- que ha significado nues- 
tra atención cuasi filosófica, pero imbuida de la conciencia que nos parece necesaria, desde las regulaciones que establece la política pública sobre la nanotecnología, es decir a las formas en que condicionamos lo que nos condiciona. En un anterior congreso de Sociología -que por sus ALAS no nos ha llevado-, dimos cuenta de una investigación realizada sobre los organismos del Estado de Chile, utilizando la Ley de Transparencia de la función pública (Ley 20.500, Chile, 2009), dónde consultamos a 24 Servicios del Estado de las regulaciones y protocolos con que se controlaba la creciente irrupción de nanotecnología en el mercado, con el resultado sorprendente (desde el punto de vista de la Política Pública), pero imaginable (desde el del ciudadano oprimido), que pese a existir amplia gama de productos que utilizan NT y NM en diferentes productos, libremente comercializados, no hay regulación, estudios sobre sus efectos, conocimiento, ni mención ninguna a la nanotecnología, en el Estado. Señalamos también allí, la tan fecunda como perversa relación entre las grandes transformaciones del Antropoceno, marcadas por lo "infinitamente grande" del Cambio Climático y lo "infinitamente pequeño" de la nanotecnología, en un proyecto de captura de CO2 en el océano, en tierras indígenas en Columbia Británica, Haida Gwen, que los relacionaba marcando tendencias.

Desde junio del año pasado, advertidos por la publicación en Wikileaks de documentos que hacían público el secreto (lo que no quiere decir que por ello haya perdido su condición de tal), del Tratado Trans Pacífico y del amplio y hegemónico alcance de sus términos, que van desde establecer nuevas formas de "propiedad intelectual" a "prohibir la mención al Cambio Climático", pasando por el tratamiento de los fondos de pensiones de los trabajadores y la Salud Pública, en la doble tenaza del "secreto" y del peso irrestricto de las grandes corporaciones capitalistas, sobre los Estados nacionales, insistimos -con necesaria ingenuidad-, en investigar sobre estas dos grandes líneas -convergentes en su antinomia de lo más grande y lo más pequeño-, de Cambio Climático y Nanotecnología, en el TPP (por sus siglas en inglés) 
a través de los mecanismos legales que contempla nuestra democracia. Los resultados son patéticos. Al cabo, simplemente, desparecen los Estados frente al Capital. Al menos permítasenos rescatar desde el humanismo, la referencia a la proximidad entre ética y verdad, aunque nos instalemos en la Grecia de Syriza y no en la tierra tecnomilitar de los poderes del capitalismo pragmático, cuantitativo, imperial, prehumano.

\section{2. "Este enunciado es falso". Epiménides.}

El estudio del ser humano resultaría mucho más simple, que el de las determinaciones históricas y culturales que condicionan su biología, si no fuera que son ellas mismas las que encontramos a la base, cuando llevamos el análisis hasta sus causas últimas. El célebre humanista y antropólogo francés Claude Lévi-Strauss, nos ha puesto en presencia de la asociación cognitiva entre el secreto y el olvido por una parte y su contrapartida complementaria, la maledicencia y la mentira, por el otro. Ambos casos corresponden a las dimensiones de una "comunicación fallida": deprimida la una, exacerbada la otra, se constituirían como una interrupción de los canales de comprensión y transmisión del intento humano por aprehender la realidad Levi-Strauss (1958)

Y si estas dimensiones remiten a las estructuras perceptibles de nuestro comportamiento como personas, mucho más importantes resultan cuando consideramos sus proyecciones en el espacio social y la magnitud política de sus alcances, como sociedades.

Hace más de un año ya, la presidenta de Brasil, haciéndose voz de la más amplia comunidad internacional, manifestó en la Asamblea General de UN, su repudio a las prácticas de espionaje masivo que por intermedio de la National Security Agency (NSA) realiza el Gobierno de los USA, sobre todos nosotros -unos más, unos menos-, toda la población del mundo. Las denuncias he- 
chas por el ex agente de la CIA Edward Snowden, habían llegado a Naciones Unidas. Y pese a que ha seguido habiendo nuevos informes de la violación sistemática de nuestros derechos humanos a la privacidad desde entonces, allí se quedaron. Simultáneamente, es cierto, ése silencio se ha hecho eco en las guerras de diversos lugares del mundo. Todas ellas generadas por miserables intereses económicos.

En Chile, el gobierno de la presidenta Bachelet, parece ajustar su programa de derechos a una concepción de la participación ciudadana que compartimos. Aún cuando no pueda haber pasos definitivos en el plano de la retroalimentación, mientras el monopolio de los media imponga la Agenda sobre la opinión pública y haya muchos espacios oscuros, creemos que desde el actual gobierno se trata de hacer claridad y profundizar la democracia, que es en definitiva, participación. Asi las decisiones sobre la Reforma Tributaria, por ejemplo, no hayan contado con ninguna retroalimentación de la gente, no cabe duda que en otros planos programáticos se han hecho esfuerzos consecuentes por que, más allá de los muros con que la dictadura cerró las alamedas por dónde pasara el pueblo y la Constitución militar siga pavoneándose en la oscuridad, con sus quórums y artilugios de terror en cámaras y pasillos, la participación popular como horizonte sustentable de la política pública, sea un hecho.

Así con la Reforma de la Educación, cuyo ciclo de diseño, ha visto el desarrollo de una variedad de instancias de convocatoria, en una búsqueda consecuente e innovadora de generar formas de participación ciudadana, o sin ir muy lejos, con el reciente anuncio de "ventanillas móviles" de SERNAC, para recoger denuncias de los consumidores contra los cobros indebidos en que la "libre empresa" y los sistemas crediticios los sumergen día a día. En éste mismo sentido, no podemos dejar de mencionar el intento de someter a Consulta de los Pueblos Originarios la creación del Ministerio de Asuntos Indígenas, como lo establece el Convenio 169 de la OIT y los Pueblos Originarios de Chile lo demandan. 
De lo anterior, que resulte tanto más sorprendente que las tratativas y negociaciones para establecer acuerdos de "Libre Comercio", con sus efectos transversales sobre todos los aspectos de la economía y la política pública, se mantengan rigurosamente fuera del alcance de nuestro conocimiento ciudadano y que haya que apelar a las "filtraciones", para saber algo.

A principios de julio, advertidos por publicaciones -entonces ya no más "secretas"-, publicadas en el sitio web de WikiLeaks y por masivas movilizaciones populares exigiendo transparencia y rechazando el TTP en distintos países (Japón, Australia, Canadá...), también en Chile distintas organizaciones han presentado cartas a Su Excelencia, la Presidente de La República, pidiendo a nombre del pueblo de Chile, se hicieran conocidos los términos del Acuerdo de Asociación Transpacífico o TPP (Trans Pacific Partnership).

La carta, pedía precisiones sobre los alcances del TTP sobre aspectos que pudieran afectar negativamente los acuerdos ya suscritos en los TLC, fundamentalmente en lo relacionado con temas de propiedad intelectual, regulaciones de internet y asuntos medioambientales. Pero no se hacía allí mención a los procedimientos de secrecía y manejo reservado de la información, que ciertamente son de la máxima importancia, particularmente cuando el sistema capitalista mundial parece entrar definitivamente en una fase de desregulación completa del que estos procedimientos -al hacerse conocidos-, dan cuenta.

El presente escrito tiene por objeto recordar que el pueblo de Chile (como todos los pueblos del mundo, en democracia), tiene derecho soberano a conocer los acuerdos "comerciales" que se hacen en su nombre y a participar de los términos y contenidos que contengan. Por otra parte, señalamos brevemente, que ésta práctica de "secretismo" de información, esconde a su vez un momento particularmente agudo de desregulación global del actual sistema de acumulación capitalista, que como nunca an- 
tes concentra el poder de decisión política y económica y el futuro del planeta en las manos de muy pocos y de -como es el caso que denunciamos- de las corporaciones sobre los gobiernos. Esta amenaza hecha realidad, se hace particularmente presente en los tratados comerciales que se están negociando secretamente y que según podemos saber por las "filtraciones" que de ellos se producen, son cada vez más atentatorios a la transparencia y permiten menos capacidades de defensa a la ciudadanía y sus legislaciones nacionales. De hecho, las posiciones más críticas, lúcidas y activas (J.Assange, S.Zizek, Y.Varoufakis), nos muestran que una vez que los TTP, TISA y TTIP entren en plena operación, los Estados perderán toda soberanía frente a las Grandes Corporaciones. http://youtube.be/Rw7PORGZQxQ(TheWikiLeaksChannel).

\section{El retumbante eco del secreto}

Precisamente, la reciente publicación de documentos hechos públicos por WikiLeaks, el Trade In Services Agreement (TISA) que concierne los Servicios Financieros, sistemas monetarios, bancos y aseguradoras, pero que también incluye "las actividades que forman parte de los Sistemas de Seguridad Social y de los planes de jubilación y retiro", es una muestra tan actual como extrema, de esta tendencia a privar a las personas de manera absoluta del conocimiento de temas que los afectan y cuyas consecuencias serán en muchos casos vitales.

En efecto, en el TISA no solo se toman las decisiones de manera inconsulta y a espaldas de las ciudadanías, sino establece que los acuerdos habrán de mantenerse secretos hasta después de transcurridos cinco años de su entrada en vigencia, lo cual sería simplemente absurdo (por cuanto los ordenamientos jurídicos nacionales han de contemplar los mecanismos a través de los cuales se implementa), si no manifestara la lógica misma de una racionalidad autoritaria, que pone a la ganancia sobre cualquier ética humana y a las empresas multinacionales sobre los 
Estados y sus jurisdicciones nacionales. Estos “cinco años” de secreto luego de su entrada en vigencia, ratifican la tendencia al aumento de secreto: el "super secreto TTPA" -sobre el que realizamos consultas-, consideraba "sólo" 4 años de secreto...

Digamos que éste acuerdo TISA, sobre el cual se tiene muy poca información (...), y que ha iniciado sus rondas de negociación durante el fin de la administración anterior, en junio del 2013, pretende liberalizar los servicios entre los mayores proveedores de servicios del mundo, está asimismo siendo negociado fuera del marco de la OMC, aun cuando muchos de sus acuerdos (todos) repercutirán sobre ella, generando un nuevo y genuino campo de inquietudes.

Sin embargo, no hay ámbito de acumulación más sensible a la protección de los Estados, y más en la mira del capital, que el de la explotación medioambiental en busca de recursos no renovables. Los distintos esfuerzos de la comunidad internacional para establecer leyes y principios que pudieran protegerlo, se han demostrado hasta aquí incapaces de establecer acuerdos vinculantes basados en criterios científicos, para lograr un desarrollo sustentable. El ejemplo más dramático de ello, se encuentra en el fracaso del Protocolo de Kioto (1997-2012), para limitar las emisiones de Gases Efecto Invernadero e impedir un recalentamiento de $2^{\circ} \mathrm{C}$ de promedio global hacia el 2030, temperatura considerada irreversible y de efectos desastrosos e incalculables para la vida en el planeta.

Y es que detrás de estas decisiones en que priman los intereses monetarios y a corto plazo, hay también una concepción inmediatista y de mercado de la política, que desconoce la necesidad de apoyar la toma de decisiones sobre criterios científicos, que permitan la necesaria regulación de áreas emergentes del conocimiento, que la "industria" ya comercializa y cuyas consecuencias se desconocen. En éste sentido, las investigaciones y el uso comercial (desregulado) de las nuevas tecnologías en general y 
particularmente de la Nanotecnología, recibirán un enorme impulso, con la venia de los organismos especializados de UN (CMNUCC), en los años a venir.

Consideremos algunas en materias tan diversas -y transcendentes-, directamente involucradas en este ejercicio de "secretos", como la geoingeniería como "herramienta" para revertir los efectos cada vez mayores del Cambio Climático, los avances en ingeniería genética, y los OGM en la agricultura, la nanotecnología y los sistemas de manejo de "Big Data" en comunicaciones. Como hemos sabido desde el año pasado, con la fuga del ex agente de la CIA E. Snowden -ya mencionado- a China y luego a Rusia, el espionaje masivo que a todos relaciona, relega nuestros derechos a la privacidad personal a un pasado mítico. Como lo señalan en su artículo homónimo Baveye y Charlet (2014), del Instituto de Ciencias de la Tierra, de la Universidad de Grenoble: Los acuerdos de Comercio, harán que las implicaciones de investigación sobre el ambiente, sean completamente irrelevantes para la toma política de decisiones.

Y es que lo anterior se hace evidente con la cláusula secreta del TTP que solicitamos precisar y buscamos clarificar, relativa a la Resolución de Conflictos entre Inversionistas y el Estado, o en inglés: Investor State Dispute Resolution, (ISDR). En efecto, dicha cláusula permitiría a corporaciones individuales, que tuvieran sus "casas centrales" en cualquiera de los países firmantes, demandar directamente a los Estados, si sus leyes infringieran sus ganancias "actuales o potenciales". Tribunales arbitrales especiales, secretos, formados por tres personas, constituidos ad-hoc, fuera de la jurisdicción de cualquiera de los países signatarios del TTP, serán encargados de arbitrar sobre cada caso y de fijar los montos y compensaciones que les parezcan.

No nos parece que el mundo -ni Chile- deban poner el futuro de sus hijos y el presente de sus mayores, en las manos de un sistema que implique que no contento con saquear las riquezas de 
tod@s, tenga además la facultad de demandar por "pagos extraordinarios" en la eventualidad que tuviéramos leyes y sistemas regulatorios que protegieran nuestros recursos. El Estado debe pronunciarse, la ciudadanía debe participar en la toma de decisiones, debemos fortalecer las condiciones para un desarrollo sustentable y equitable y no lo contrario.

\section{Matar por los hidrocarburos fósiles.}

Y es que si lo dicho parece lejano y es..."secreto", lo cierto es que la desregulación ha llegado hasta la guerra y es perfectamente actual. La que se hace a distancia, con vehículos voladores no tripulados, o Drones. Asesinato a distancia, sin que haya sistema jurídico ni normativo alguno, más que la orden militar que lo autoriza. Hace un par de semanas, el presidente de los USA acaba de autorizar la utilización de bombardeos con Drones sobre el territorio del actual así proclamado Estado Islámico (EI), territorios que antes de la invasión USA, y su posterior retiro, conocíamos como Irak y Siria. En un estudio reciente de una ONG Alemana, se calculaba en alrededor de 3.500 los muertos en Pakistán y Afganistán, gracias al uso de esta revolucionaria técnica de exterminio selectivo (o no tanto...). Allí mismo se calculaba en un $2 \%$ los muertos de quienes efectivamente pertenecieran a los grupos "terroristas" y así parezcan pocos los 70 muertos implicados, frente a los 3.430 (campesinos, niños, ancianos) que no tenían arte ni parte, eso quiere decir también -y tendemos a olvidarlo-, que 70 personas fueron asesinadas sin juicio ninguno, en países lejanos, a los cuales no se les ha declarado la guerra, ni se les ha consultado por autorización ninguna, por profesar ideas contrarias al poder y que 3,430 fueron asesinadas, porque sí.

Lamentablemente esta "desregulación de la guerra", que en parte importante se apoya en la "externalización" de los "servicios", para favorecer intereses de inversionistas privados, no es un he- 
cho aislado. El reciente Genocidio de Israel sobre la franja de Gaza, y la enorme proporción de mujeres y niños asesinados en sus casas, o en las escuelas en que se refugiaban, debe indicar una proporción similar de masacres civiles y de víctimas inocentes, que no tienen nada que ver. Salvo tal vez, habitar en esos lugares, entonces, malditos. Aun cuando en este último caso, ha habido múltiples decisiones condenatorias de organismos competentes que se han ignorado. Allá como acá, lo que se pueda saber, resulta cada vez más más difícil, cuanto que los poderes se concentran en las mismas manos de los cada vez menos, que obtienen los exclusivos privilegios y controlan las fuentes de acceso a la información.

5. ¿Para quién los "beneficios" y de que estamos hablando? En reciente entrevista a los media (www.democracynow.org), el Premio Nobel de Economía Joseph Stiglitz, señalaba que los Tratados de Libre Comercio, se habían centrado hasta ahora, en las bajas de tarifas, para generar incentivos de competencia y mayor acceso de países y personas al consumo. Considerando las áreas que han resistido a éste esquema de liberalización y que por implicar esferas directamente políticas, son llamadas "áreas sensibles", señala, la nueva serie de tratados (TTIP, TISA...) que sobre la base del TTP se modelan, no estarán orientados a lograr mayores bajas de tarifas, sino a disminuir los estándares de sus procesos regulatorios. Esto es, disminuir la incidencia de los cuerpos legales que frente a la codicia ciega de las corporaciones, protegen (protegían...), -o estaban concebidos para proteger-, al medioambiente, los consumidores, los trabajadores, la salud. En otras palabras, las grandes corporaciones, que bajo la égida política de los USA (la economía más importante de las 12 actualmente incorporadas al TTP), se articulan en éstos acuerdos, pretenden dar un paso definitivo en aumentar sus ganancias a costa del bienestar real de las personas.

Si seguimos el razonamiento del citado Stiglitz, estos "acuerdos" no tienen nada que ver con el "comercio", sino con los principios 
que los regulan. Si nos permitimos la metáfora, no son sobre la eficiencia energética de los vehículos, sino sobre las leyes del tránsito. 0 más bien - y es el punto - sobre su desaparición definitiva. Como las leyes son nacionales y el sistema financiero no lo es, la desregulación significa que las empresas transnacionales adquieren de manera definitiva, más poder que los estados. Consignemos que en la actualidad de las 100 economías más importantes del mundo, 40 son ya corporaciones y la tendencia es, claro, a que sigan aumentando en desmedro del poder de los Estados nacionales. Sobre todo, si aceptamos que instancias de "resolución de conflictos" como las contempladas en estos "tratados" y ya activas y operacionales desde hace unos años en el Banco Mundial, se lleven a cabo.

Es así que sobre la base de esta "avanzada nueva ética" de regulación, se haya iniciado una serie de demandas contra los estados nacionales. Por ejemplo, la compañía de cigarrillos Philip Morris, está demandando al Uruguay por haber iniciado una campaña gubernamental en contra del consumo de cigarrillos, por sus conocidos efectos sobre la salud de las personas, aduciendo que su política interfiere con sus derechos comerciales. Es decir el derecho del "libre comercio", de matar personas.

De acuerdo a las condiciones de generación de instancias jurídicas ad hoc, que establecen éstos tratados para la "resolución de conflictos", no puede caber duda que Uruguay -además de asumir como Estado, los ingentes costos en salud pública-, habrá de pagar millonarias “compensaciones” a la Compañía Tabacalera, por tratar de impedir que comercialice el veneno entre su población. La decisión de una corte de Nueva York, anulando previa decisión de Tribunales, que obligaba a la Compañía petrolera Chevron a pagar al Estado Ecuatoriano cerca de US\$ M 8.000 en reparaciones por un desastre medioambiental y estableciendo por el contrario que es el Estado de Ecuador quien debe pagar cerca de US\$ M 2.000 a la petrolera, puede ser paradigmático de lo que decimos. El Salvador, Bolivia, Perú y Argentina han sido 
objeto de estas muy paradojales resoluciones de "justicia", que los nuevos "tratados" llevan al paroxismo del absurdo y que se negocia en el máximo secreto.

En realidad, estas cláusulas secretas a la resolución de conflictos, contenidas en el TTP y los demás, son el capítulo final de un libro que se viene escribiendo hace ya varios años: al alero del Banco Mundial y su tribunal de Resolución de Conflictos. A la fecha se han presentado más de 100 demandas contra los Estados, en tribunales especiales, "independientes", compuestos por tres miembros y que tienen el poder de decidir sobre los Estados y sus legislaciones. De seguir su hasta aquí promisorio camino, los Tratados "económicos" y sus cláusulas secretas, es esperable que las demandas de las corporaciones a los Estados aumentarán continuamente, gravando así aún más a las naciones en desarrollo, que ya ven disminuir exponencialmente sus recursos naturales y la pérdida de la biodiversidad, en favor de la concentración de capital en manos de las grandes corporaciones.

\section{Allegro e Finale (ma non troppo)}

Tres reflexiones finales para abrir el debate entre nosotros. La primera tiene que ver con que el poder de las corporaciones transnacionales, se impone como nunca antes sobre los gobiernos democráticos, vulnerando las legislaciones con que -mal que bien-, los países protegían a sus ciudadanos. ¿Cómo puedo estudiar los efectos de la nanotecnología en los sistemas alimentarios, si no se me permite conocer los términos ni condiciones de un marco superior que los condiciona, regula, determina e ignora?.

La segunda es que probablemente al vincular estos procesos con la guerra (a distancia) y los mecanismos abiertamente ilegales con que esta se ejerce en la actualidad, no estemos en rigor en presencia de nada nuevo: cuando el gobierno de Inglaterra -con- 
tando con el pronto y atento apoyo- de los gobiernos de occidente (USA, Francia, Alemania... Rusia), inició la Guerra del Opio contra China hace 150 años, porque su gobierno se opuso a la masiva comercialización de opio entre su población por los ingleses, la razón también fue la misma: China se estaba oponiendo a los "derechos básicos del comercio".

La tercera, es que no podemos seguir aceptando -cualquiera que fueren nuestras posiciones-, que decisiones de tanta importancia sobre el futuro del país y sus generaciones, sea tomado por personeros de gobierno, en medio de procesos ininformados que no vinculan a la población y cuyas condiciones de secrecía, vulneran nuestras propias disposiciones constitucionales y legales vigentes. En efecto, el Articulo 8 de la Constitución chilena, sancionado por La Ley 20.283 y refrendado por Reglamento, señala que "todo acto realizado por la administración debe ser público a no ser que este expresamente protegido por una Ley Especial de Quórum Reservado". Ello, ciertamente se contradice con que oficiales del Gobierno, acepten y autoricen la firma de documentos "secretos", como los Tratados que señalamos, aun cuando éstos se encontraran en fase de negociación.

Desde aquí tendríamos que llamar simultáneamente la atención sobre la tendencia brutal, veloz, inmediata y además secreta, de la tendencia hegemónica de las formas de acumulación capitalista hacia la desregulación y considerar la relevancia global de acuerdos legales vinculantes, que contemplen aproximaciones transnacionales para evitar las guerras, permitirnos efectivamente la adaptación al cambio climático, la introducción de nuevas tecnologías, la protección de nuestros recursos naturales, la conservación de la biodiversidad, los océanos y la criósfera, por sobre los intereses de las grandes corporaciones. Ello supone ponernos en presencia activa de las convenciones existentes y sus marcos legales y avanzar en las categorías necesarias de implementación. 
Sin duda que instrumentos como los definidos por la Convención de Aarhus sobre el acceso a la información, la participación pública en la toma de decisiones y el acceso a la justicia en asuntos ambientales, la decidida voluntad política de participación sistémica en las COP20 de Lima en diciembre y COP21 en Paris el 2015, en la Convención Marco de Cambio Climático de Naciones Unidas (CMNUCC), de acuerdo a criterios éticos, más allá de los intereses que dictamina el mercado, el trabajo intergubernamental, con plena participación ciudadana que requieren las propuestas de Río+20, las Metas Sustentables del Milenio (SDG), y la Convención sobre la Bio Diversidad (CBD), habrían de generar las condiciones sociales adecuadas, para empezar a superar el oscurantismo que se nos impone y crece.

Así, otra vez, hemos sido impedidos de conocer de qué manera se prefigura la legalidad de la nanotecnología en el espacio público. Rescatamos el carácter semántico del ejercicio: no hay fiesta lúdica en éste ejercicio tramposo de expropiación de derechos soberanos, ni ha elegido nadie sus afinidades, en esta sensación de vulneración de derechos que nos utilizan como parte de una comparsa que sirve los intereses espurios de quienes han puesto al Estado al servicio del capital: Tampoco refiere al ámbito reducido del grupo de personas que fuéramos, sino a la noción simbólica y trascendente de un pueblo todo. No es juego, -sino tal vez "de guerra"-, el que un instrumento del Capital y el mercado internacionales, se imponga sobre los principios regulatorios del estado nación. Es, por el contrario de una importancia indesmentible y que no puede ser ignorada, pero el que este "tratado" se permita "prohibir" que se haga mención al Cambio Climático, es decir el mayor desafío de política pública de los países del mundo, francamente inaceptable. Mucho más cuanto que cada día se multiplican los estudios de la magnitud de su impacto ya presente, en las más diversas dimensiones de la vida sobre el planeta. Vale citar en éste sentido, el informe de los estudios del impresionante impacto del Cambio Climático en Salud, realizados por la Comisión Internacional de médicos de diferentes especialidades, publicado en ju- 
nio en la prestigiosa publicación inglesa The Lancet, informes del PNUD y CEPAL que establecen de manera inequívoca la relación entre Cambio Climático, inequidad y pobreza, la Encíclica papal, que se hace cargo de la evidencia científica y advierte de la necesidad de una "verdadera revolución cultural" para cambiar los principios del capitalismo imperante, que entrega todo el poder a los bancos y nada a las personas ni sus principios.

El secreto se opone a lo público, como la noche al día. Y si la contradicción explicaría su existencia, así como -hasta aquí-, no puede haber hombres sin mujeres (y viceversa), es característica de la época y de sus formas de objetivación finales, el que estos opuestos se complementen en el explosivo espacio de su irreductible antinomia. También, que el secreto y la mentira forman parte del mismo espacio en que se altera el diálogo y su camino a la verdad. Esa que, también es paradoja, tod@s necesitamos para ser.

Y es que:

A. No puede haber "negociación" ninguna que sea secreta, es decir, a espaldas del pueblo soberano. Esa es nuestra política.

B. Éste "tratado" que se nos impone, no es un tratado más de "libre comercio" (STIGLITZ, 2014), sino un arreglo para comprometerse a terminar con los Estados en beneficio de las Grandes Corporaciones (que son las que -a través de Obama-, dictan sus términos y alinean marionetas). Esta es nuestra economía.

C. Es absolutamente delirante (¿hasta dónde puede llegar la ignominia?) y de ninguna manera aceptable, -por nadie-, que una dependencia del Ministerio de Relaciones Exteriores, (Direcon, Chile), diga que "no reconoce la Convención Marco de Naciones Unidas sobre Cambio Climático, ni sus instrumentos" ${ }^{2}$... Ésta es nuestra ciencia.

2 http://www.direcon.gob.cl/wp-content/uploads/2014/04/Cuarto-Adjunto-Medio-Ambiente-20140526.pdf 


\section{N ostos (griego: H istoria de retorno a casa). A guisa de Conclusión hacia delante.}

La nanotecnología nos muestra que la materia, tiene propiedades distintas según sea la escala en que la estamos considerando. Esto ha sido varias veces señalado en este mismo Seminario de Renanosoma, así que no insistiré, ya que mi reflexión, por otra parte, apela al juego de las ideas. En efecto, como nos dice Levi-Strauss, "una hipótesis puede tener un valor operacional efectivo, sin que la evolución posterior se deba a probar en ella la verdad última de lo real". Decimos nosotros y puede parecer obvio, que también los espacios sociales y las personas que por ellos son conformadas al tiempo que los transforman, pueden adquirir diversas propiedades de conciencia y asociación, y que ellas no necesariamente sean entendibles desde los mecanismos de control ni las visiones tradicionales. La observación de las propiedades de la nano, podría ayudarnos a entenderlo y potenciar la búsqueda de maneras completamente nuevas de oposición y resistencia a la guerra del capitalismo contra el planeta. Al cabo, de muchas maneras, estamos viviendo lo que en términos de una filosofía de la historia, podríamos llamar Renacimiento. Dotado de las características ya no contradictorias, sino antinómicas de éste Antropoceno, cuyos procesos de acumulación del Capital (y el poder) en manos de tan pocos, como hace tan sólo unos años era impensable, vino acompañado de un prodigioso desarrollo tecnológico, que produjo pérdidas ya irreversibles en todos los planos de expresión de la vida en el planeta, y dónde las clases que pugnaban por controlar los procesos, dieron paso a una verdadera sociedad de castas, que tampoco dialogan entre sí, como se esperaría que ocurriera en cualquier democracia. Como nos enseñó T. Kuhn (2004) en Las Revoluciones Científicas, los cambios de paradigma se producen de maneras que sólo son comprendidos (y probablemente perceptibles) una vez que ya han ocurrido. Como en la Revolución Copernicana, que al cuestionar el sistema Ptolemaico que hacía girar al sol y los planetas alrededor de la tierra, puso al ser humano en el centro y a la naturaleza afuera, con la Revolución Industrial y la tecno-ciencia, al servicio del capital, nos 
damos cuenta que el Antropoceno, al actuar de manera global, simultáneamente sobre lo más grande (la atmósfera que nos rodea) y lo más pequeño, (la nanotecnología), ha invertido esa figura de la revolución copernicana, poniendo a la naturaleza en el centro de la cultura. Habremos de volver.

\section{Referências}

BAVEYE, P. C. and CHARLET, L. Proposed Trade Agreements Would Make Policy Implications of Environmental Research Entirely Irrelevant. Environmental Science \& Technology, 48 (3), 2014.

DEBRAY, Regis. Introduction a la Mediologie. Presses Universitaires de France, PUF, Paris, 2000.

KHUN, Thomas S. Las Revoluciones Científicas. Fondo de Cultura Económica, FCE, Buenos Aires, 2004.

LEVI-STRAUSS, Claude. Anthropologie Structurale. Plon: Paris, 1958.

MARX, Carlos. El Capital. Crítica de la Economía Política. Argentina: Editorial Cartago, 1973. Tomo I.

Trade in Services Agreement (TISA) Financial Services Annex. WikiLeaks release: June 19. Disponível em www.wikileaks.org,_ 2014.

\section{URL}

http://www.democracynow.org

http://es.justinvestment.org/

http://www.citizenstrade.org/ctc/

http://www.canadians.org/trade/issues/TPP/index.html

http://www.foe.org/projects/economics-for-the-earth/trade/trans-pacific-partnership

http://justinvestment.org/2013/02/peril-in-the-pacific-no-to-tpp/

http://justinvestment.org/2014/08/ttip-poder-ilimitado-a-las-multinacionales

http://www.truthdig.com/avbooth/item/michael_hudson_the_tpp_and_europes_central_bank_against_syriza_20150528\#.VXTcHGwcGYg.mailto http://www.kineikon.blogspot.cl/ (Simulacros."www.hechosocialtotal.antropología")

Recebido em 03/03/2016

Aprovado em 05/05/2016 


\section{Anexo}

\section{Respuesta de consulta al Ministerio de Relaciones Exteriores de Chile, sobre el Trans Pacific Partnership (TPP).}

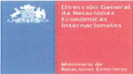

DENIEGA SOLICITUD DE ACCESO DE INFORMACIÓN LEY NO $20.285(C-107)$

SANTIAGO, 28 de octubre de 2014

\section{Resolución Exenta J-1078}

VISTOS:

El artículo $8^{\circ}$ de la Constitución Política de la República; la Ley 20.285, de transparencia de la función pública y acceso a la información de la transparencia de la función pública y acceso a la información de la Administración; el Decreto del Ministerio Secretaría General de la Presidencia No13 de 2009, Reglamento de la Ley No 20.285; el Decreto con Fuerza de Ley No 53 del Ministerio de Relaciones Exteriores, de 1979, que crea la Dirección General de Relaciones Económicas Internacionales del Ministerio de Relaciones Exteriores y establece su estatuto orgánico; la Resolución de la Dirección General de Relaclones Económicas Internacionales, Exenta No 1-204, de 2009, que establece las reglas de procedimiento aplicables a las solicitudes de acceso a información recibidas en el Servicio; el Memorándum No 7.771, de 24 de octubre de 2014, de la Dirección de Asuntos Económicos Bilaterales; el Decreto Supremo $N^{\circ} 41$ del Ministerio de Relaciones Exteriores, de 2014; y la Resolución de la Contraloría General de la República No 1.600, de 2008, que fija normas sobre exención del trámite de toma de razón.

\section{CONSIDERANDO:}

1.- Que el artículo $8^{\circ}$ de la Constitución Política de la República establece que son públicos los actos y resoluciones de los órganos del Estado, así como sus son publcos los actos y resoluclons fundamentos y los procedimientos que utilcen y que solo una ley de quorum

calificado podrá establecer la reserva o secreto de aquéllos o de éstos, Dirger 
Rainer María Hauser Molina

dichos órganos, los derechos de las personas, la seguridad de la Nación o el interés nacional.

2.- Que el artículo 5 de la Ley No 20.285 dispone que, en virtud del principio de transparencia de la función pública, los actos y resoluciones de los órganos de la Administración del Estado, sus fundamentos, los documentos que les sirvan de sustento o complemento directo $y$ esencial, $y$ los procedimientos que se utilicen para su dictación, son públicos, salvo las excepciones que establece esta ley y las previstas en otras leyes de quórum calificado.

3.- Que, el artículo 14 de la citada Ley señala que el jefe superior del servicio requerido deberá pronunciarse sobre la sollcitud, sea entregando la requerido debera pronunciarse sobre la sollcitud, sea entregando la información solicitada o negándose a ello, en un plazo máximo de veinte dias Decreto del Ministerio Secretaria General de la presidencia vo13 de 2009 , Reglato del Ministerio Secretaría General de la Presidencia No 13 de 2009 , Reglamento de la Ley No 20.285 , dispone que en caso que el órgano
servicio requerido deniegue la solicitud de información en virtud de alguna de las causales de secreto o reserva que establece la ley, deberá formular su negativa por escrito, fundada y por el medio que corresponda.

4.- Que, de conformidad con lo dispuesto en el artículo 21 NO1 de la citada Ley No 20.285, constituye una causal de secreto o reserva en cuya virtud se podrá denegar total o parcialmente el acceso a la información, cuando su publicidad, comunicación o conocimiento afecte el debido cumplimiento de las funciones del órgano requerido.

5.- Que, de conformidad con lo dispuesto en el artículo 21 No4 de la Ley No 20.285, constituye una causal de secreto a reserva en cuya virtud se podrá denegar total o parclalmente el acceso a la información, "cuando su publicidad, comunicación o conocimiento afecte el interés nacional, en especial si se refieren a la salud pública o las relaciones internacionales y los intereses económicos o comerciales del pais".

6.- Que por solicitud de acceso a información, folio No AC002C-0000107, formuladar por formulada por el peticionario don que chile se encuentra negociando".

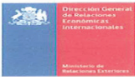

7.- Que, por el Memorándum No 7.771, de 24 de octubre de 2014, la Directora de Asuntos Económicos Bilaterales subrogante manifiesta la imposibilidad de entregar la información requerida relativa a TPP y TiSA, ya que la misma hace referencla directa a los textos de negociación propuestos que la misma hace referencla directa a los textos de negociación propuestos por Chile y demas paises participantes en el marco de la negociacion, huestro pais en el marco de la negociación de Tisa.

8.- Que, en el marco de las negociaciones del Tratado Transpacífico (TPP, por sus siglas en inglés). Australia, Brunel, Chlle, Estados Unidos, Malasia, Nueva zelandia, Perú, Singapur y Vietnam hón llevado a cabo numerosas rondas de negoclaciones, entre grupos gubernamentales que discuten diversas materias, tales como Inversiones, Acceso a Mercados, Servicios, Asuntos Legales, Aduanas, Propiedad Intelectual, Cooperación, Medio Ambiente, Movilidad de Personas, Servicios Financieros, Reglas de Origen, Medidas Sanitarias y Fitosanitarias, Obstáculos Técnicos al Comercio, Compras Públicas, Competencia, Asuntos Laborales, Temas Horizontales y Comercio Electrónico, entre otros.

9.- Que el objetivo del Gobierno de Chile en las negociaciones del TPP consiste en la creación de una zona de libre comercio que integre a las economías de Asia Pacífico, la que representa actualmente más de un $45 \%$ del total del comercio que reallza nuestro país con el mundo. Chile, que es socio activo de APEC, cuenta con acuerdos bilaterales vigentes con Australla, Grunei Darussalam, China, Corea, India, Japón, Molasia, Nueva Zelandia, Vietnam y Singapur.

10.- Que, aceptar el requerimiento formulado implicaría entregar los textos presentacios por los gobiernos de los países participantes en esta negociación internacional el debido cumplimiento de las funciones del Servicio y el interés nacional, ya que se refieren a las relaciones internacionales del país.

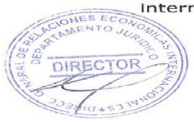


11. - Que las propuestas de texto han sido intercambiadas entre los países participantes de la negociación del TPP en un proceso de comunicación recíproca, con la seguridad de que se mantendrá su confidencialidad, y gobierno chileno, dafiando la capacidad negociadora de Chile y sus relaciones exteriores con los demás países participantes en la negociación, lo que sin duda afectaría el interés nacional. En efecto indudablemente si como resultado de lo anterior se frustrara el objeto de la referida negoclación, cual es, la creación de una zona de libre comercio que integre a las economías de Asla paciol zona que concentra internacional de nuestro país con el mundo, ello significaría una grave

12.- Que, en virtud de lo anterior, es dable concluir que la entrega de la información requerida sobre el Tratado Transpacífico no sólo está impedido por el compromiso con los demás países participantes de dicha negociación, sino que ello podría afectar el debido cumplimiento de las funciones de esta Dirección General, frustrando el proceso de negociaciones en curso, ya que su conocimiento y difusión pública puede afectar la estrategia de negociación en contra de los propios intereses chilenos.

13.- Que, atendido el alto desarrollo y potencial de comercio internacional en el sector de Servicios, que según la Organización Mundial del Comercio (OMC) genera las dos terceras partes del valor total agregado mundial y sus exportaciones crecen a tasas de más del $10 \%$ en todo el mundo, un grupo de economías en el marco de la OMC, decidió, en el 2011, iniciar conversaciones destinadas a negociar un acuerdo plurilateral de servicios denominado Trade in Services Agreement (TISA). Actualmente participan en este proceso de negociación 51 economías, entre ellas la Unión Europea que está compuesta por 28 países, que en conjunto equivalen aproximadamente a un $75 \%$ del comercio mundial de servicios.

14.- Que los países participantes en TISA acordaron administrar de manera confidencial los textos consolidados del proceso de negociación y las propuestas elaboradas por los demás países. No obstante, no lograron acordar una cláusula específica que regulara esta materia dada la acordar una cláusula específica que regulara esta materia dada la uatticipantes.

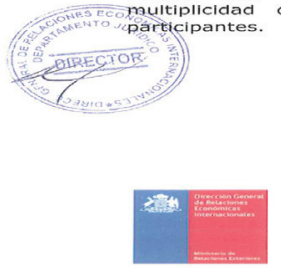

15.- Que, no habléndose logrado consensuar la propuesta de entendimiento formulada en marzo de 2013 por la delegación de Estados Unidos, ésta requirió a las demás que señalaran los regímenes nacionales en materia de confidencialidad para proteger los documentos relacionados a la negociación de TiSA. En respuesta, Australia, Canadá, Colombia, Corea, Estados Unidos, unión Europea méxico Noruega, Panamá Taíá Chino y Turquía há Unión Europea, Mexico, Noruega, Panana, Talpế Chino "Y Turqua han "reservado" aplicable al proceso de negociación de TiSA.

16.- Que, si bien los demás países aún no han notificado el tratamiento aplicable, todos los documentos y propuestas presentados en el marco de aplicable, todos los documentos y propuestas presentados en el marco de "Distribuciacion están marcados con la frase "Información Confidencial" o "Distribución Limitada a los Participantes en TiSA" u otras expresiones
similares. En consecuencia, no cabe duda que tales documentos y similares. En consecuencia, no cabe duda que tales documentos y
propuestas, han sido hechas bajo la expectativa cierta de que el tratamiento que los demás participantes darán a esa información será confidencial.

17.- Que esta Dirección General invariablemente ha denegado el acceso a los textos de negociación de TPP y TISA, invocando las causales de reserva previstas en los numerales 1 y 4 del artículo 21 de la citada Ley, como consta en las Resoluciones Exentas del Servicio No 3-294/2012, No J-377/2012 y $N^{\circ} \mathrm{J}-957 / 2014$. El fundamento de estas denegaciones fue reconocido expresamente por el Consejo para la Transparencia, en sus Decisiones de Amparo Rol C666-12 y Rol C738-12, respectivamente.

18.- Que, en la Decisión de Amparo Rol C666-12, el Consejo para la Transparencia concluye que "entre las partes que intervienen en las negocianes - incluso que "entre las partes que intervienen en las 位tía una expectativa razonable que, en relación con la documentación y propuestas que interambio respecto de los intervinientes $y$ vedarse el mismo a terceros ajenos a la negociación. En base a ello, cabe inferir que un comportamiento que suponga transgredir dicha efectuadas por las partes o a los datos suministrados por éstas a los demás 
países con el único fin de desarrollar las negociaciones - implicaría una actuación que afectaría el debido cumplimiento de las funciones de la DIRECON, en cuanto encargada de las negociaciones del TPP en representación de Chile, toda vez que habría una alta probabilidad de dafiarse su posición en dicha negociación, produciendo una pérdida en la confianza depositada en el marco de dicho tratado, todo lo cual podría poner en riesgo la celebración de nuevos acuerdos de libre comercio o el cumplimiento de aquellos ya suscritos por Chile".

19.- Que, asimismo, dicho Consejo en Decisión de Amparo Rol C738-12 concluye que existiría una expectativa probable, en caso de divulgarse los documentos solicitados-desatendiéndose, de esa forma, el acuerdo de confidencialidad - de que se produjera un perjuicio en las relaciones internacionales de chile respecto de los demás paises que intervienen en las internacionales de Chile respecto de los demás paises que intervienen en las negociaciones del TPP, "afectando, de esa forma, la posición del Estado de desarrollo, pudiendo razonablemente causarse un dano a las relaciones desarrollo, pudiendo razonablemente causarse un daño a las relaciones internacionales $\vee$ a los intereses económicos y comerciales del país". agregando que la comunicación de la información solicitada afectaría también el interés nacional, por cuanto debe estimarse reservada de la misma rorma
como fue resuelto en las decisiones de los amparos Rol C1233-11, C1234-11 como fue resuelto en las decisiones de los amparos Rol C1233-11, C1234-1
y C666-12.

20.- Que, el Consejo para la Transparencia en Decisión de Amparo Rol c1534-12, tomando como base lo establecido en las aludidas Decisiones de Amparo Rol C666-12 y Rol C738-12, reitera el valor de un acuerdo de confidencialidad para el intercambio de información entre países participantes de una negociación internacional, aunque éste carezca de fundamento normativo, lo que es plenamente aplicable en el caso de TiSA.

\section{RESUELVO:}

I. Deniéguese parcialmente la solicitud de información No AC002C-0000107, en relación con todos los textos de TPP y con aquellos textos que refleján las propuestas de negociación de los demás participantes textos que reflejan las propuestas de negociación de los demás participantes del artículo 21 de la Ley No 20.285 sobre Acceso a la Información Pública.

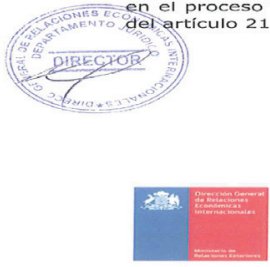

II. Entréguese copia del Memorándum No 7.771 de la Directora de propuestas de Chile en el marco de 24 de numera dicho 2014 , y de las

IIx. Notifiquese la presente resolución al peticionario don mediante correo electrónico dirigido a

IV. Incorpórese la presente resolución denegatoria en el índice de Actos Y Documentos calificados como secretos o reservados de la Dirección General y Documentos callificados como secretos o reservados de la Dirección General en conformidad a lo dispuesto en la Instrucción General No 3 del Consejo para la Transparencia.

\section{ANÓTESE, NOTIFÍUESE Y ARCHÍVESE}

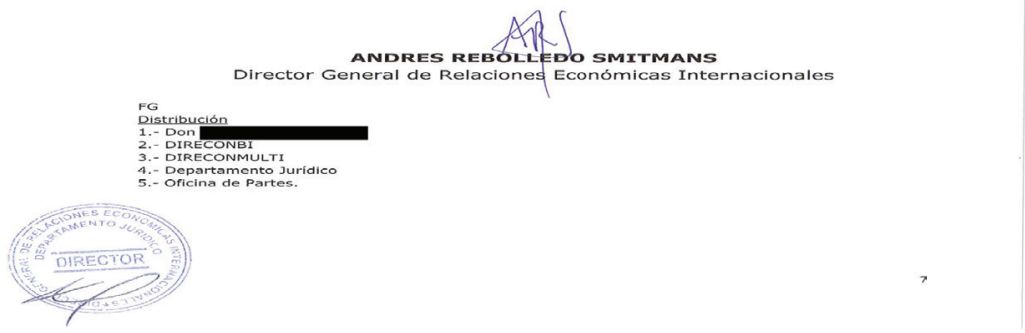


